\title{
A Falling Detection System with wireless sensor for the Elderly People Based on Ergnomics
}

\author{
Zhenhe $\mathrm{Ye}^{1}$, Ying $\mathrm{Li}^{2}$, Qiaoxiang Zhao ${ }^{2}$ and Xue $\mathrm{Liu}^{3}$ \\ ${ }^{1}$ College of Mechanical and Electrical Engineering, Agricultural University of HeBei, \\ Baoding, HeBei, 071000, China \\ ${ }^{2}$ College of Arts, Hebei Normal University of Science \& Technology, Qinhuangdao, \\ 066000, China \\ ${ }^{3}$ School of Computer Science, Harbin University of Science and Technology, Harbint, \\ HeiLongJiang, 150080, China \\ yezhenhe@163.com

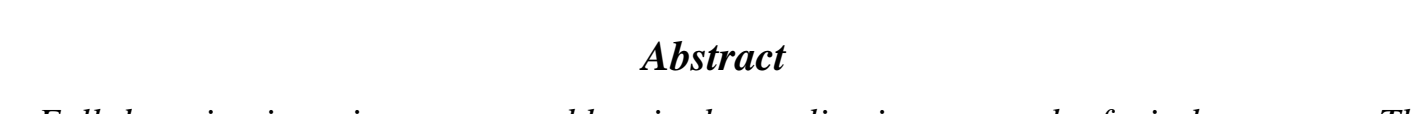

Fall detection is an important problem in the application research of wireless sensor. The paper presents wireless sensor architecture based human falling detection system especially for elderly people. The falling detection system is implemented using 3-axis acceleration sensor to measures and collects the elderly people activities acceleration and transfer data by zigbee-3G network to remote medical monitoring system platform, which makes a preprocessing method that suspected data is acquired based on one -class SVM classification algorithm. The algorithm analyzed different action which expended different threshold ranges of energy to judgment, and then analyzed the special temporal speed, displacement and angle as an auxiliary criterion for judgment. The experiments show that the application can offer a new guarantee for the elderly health.

Keywords: fall detection, tri-axial accelerometer, one-class SVM, zigbee

\section{Introduction}

Currently, the world face more aging problem. China is also aging population problem, with more and more old people and quick rising rate. According to statistics, China's elderly population aged over 60 was more than 1.86 billion in 2012, it made up14.3 percent of Chinese population. Since 1980, China's elderly population aged over 60 with an average annual rate of $3.32 \%$ growth. By 2050 society will enter an advanced stage of ageing. China's population is aging at unprecedented levels. The falling is quite common among the elderly. According to one estimate, falling had happened on 1/3 person aged over 65 every year, which will increase along with the increment of age. Falling is the leading cause of elderly aceidental injury. And it is likely to lead to fall when accidental And it is like]y to lead to fall when accidental slip or some disease attack suddenly and deterioration. If not promptly timely rescue, delay salvage timing when the fall happened will be life-threatening. Therefore, the development designed for elderly service produets-elderly fall monitoring system which can accurately differentiate between fall events and active daily life events, and can timely alarm and contact ambulance in the cases of not affect older people's normal life, it has important role in improving the life quality of elderly and promoting the stability of our society [1-4].

Nowdays, we can classify the fall detection technology in three ways from the different signal sampling: A fall detection technology - based video technology, has the weak script of limited detection range and it may disclose the personal privacy; A fall detection technology - 
based sound technology with little accuracy, is closely related with different ground and floor, A fall detection technology - based wearable sensor technology is the wearable device with microsensors, such as glasses, hats, shoes, dress and so on. It can be real-time monitoring of human activities. When body movement parameters changed, it may determine if the elderly falling down. Fall detector is more suitable for fall detection system based on wearable sensor because it not being subject to the limitations of the detection location [5-6].

This study designed a new device, which could be attached to the waist of the users, for detecting the falling of the elderly people based on the data acquisition function of the acceleration sensor. For the stage of data preprocessing, a classification algorithm based on 1class SVM was proposed. The final falling judgment was made according to the difference of the greatest energy consumption of the human body (the difference of the threshold range) in different actions. To ensure the accuracy, three additional Eigen values of the human body (velocity, shift and title angle) in the specific time domain were also included as an auxiliary judgment basis.

\section{The Design of the Falling Detection Module}

\subsection{The Overall Structure}

The overall structure of the falling detection system for the elderly people based on Zigbee is shown in the following figure [7,8]. It consists of three components: the portable module containing Zigbee nodes for falling detection the Zigbee-3G network and the remote medical monitoring system platform. The detailed network strueture is as follows: the acceleration collecting unit of the portable falling detection module collects the raw acceleration data; the microprocessor unit preprocesses the signal; the suspicious data captured during the preprocessing stage is transmitted (1a) the wireless sensor network of the remote medical monitoring device (the wireless sensor network constitutes of the nodes of the wireless Zigbee sensor, the multi-level routing nodes and the concentrator) [9]; the concentrator integrates the Zigbee-3G network and sends the data to the remote falling detection system; the data processing system of the rembte falling detection system analyzes the relevant data comprehensively. If a lalling action is confirmed, the system will automatically trigger the alarm [10, 11] The interactive unitmainly consists of the function keys, the LED indicators and the buzzer. The function keys enable the users to activate an alarm or cancel a false alarm. The LED indicators are mainly used to display the connection status of the communication network. The buzzer can obtain a feedback alarm signal when the system detects a falling.

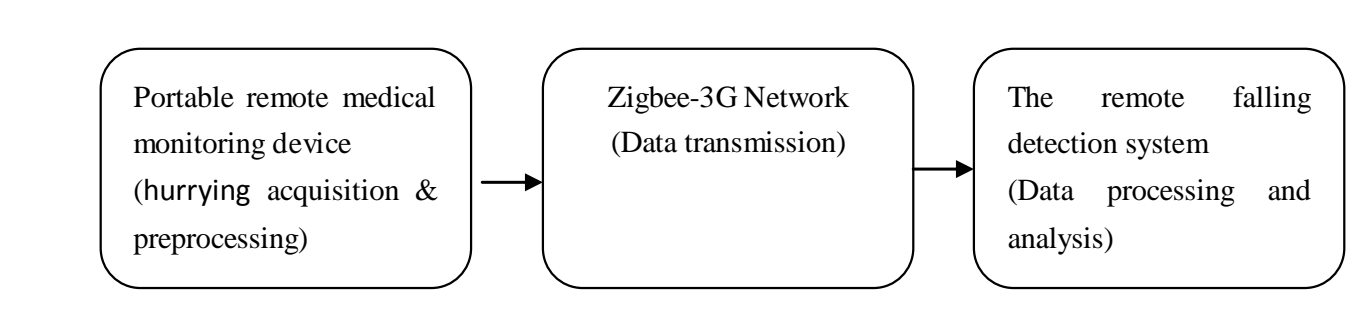

Figure 1. The Overall Structure

\subsection{The Zigbee Network}

The wireless sensor network is also known as the Zigbee network. It has a series of unique features, such as short distance, low complexity, self-organization, low power consumption, low data rate and low cost. The Zigbee network is mainly suitable for the application fields of 
automatic control and remote control. It can be embedded into a variety of devices. From the first version - ZigBee 2004 which was released in February 2004 to ZigBee 2007 (ZigBee PRO) which was released in 2007, this system has been constantly optimized in terms of network reliability, network capacity, power consumption, interference resistance and many other aspects. Comparing with the previous versions, ZigBee 2007/pro has achieved the following technical improvements:

1) ZigBee 2007/pro uses the industrial and commercial grade protocol;

2) ZigBee 2007/pro is suitable for large-scale networks (large-scale wireless sensor networks containing more than 1000 nodes);

3) ZigBee 2007/pro applies an enhanced routing method. The routing has become more reliable, and the routing table can save more memory;

4) ZigBee 2007/pro is equipped with the high-level frequency hopping technology, so it has a strong anti-interference ability;

5) ZigBee 2007/pro can transmit large-size data packet via multiple packages,

6) ZigBee 2007/pro provides the commercial grade encryption communication.

\subsection{The 3-axis Acceleration Sensor}

This study applied ADXL345 which is manufactured by ADI Company as the falling detection equipment. ADXL345 is a 3-axis acceleration sensor with digital outputs based on iMEMS technology. Its main features are as follows: multiple measuring ranges including $+/-$ $2,+/-4,+/-8$ and $+/-16 \mathrm{~g}$; highest resolution 13bit; fixed sensitivity: $4 \mathrm{mg} / \mathrm{LSB}$; super low power consumption: 40-145uA; standard I2C or SPI digital interface; Level-32 FIFO storage; multiple motion state detection; and flexible termination mode. These features can greatly simplify the falling detection algorithm, so ADXL345 is very suitable for falling detection. Its pin diagram is shown below:

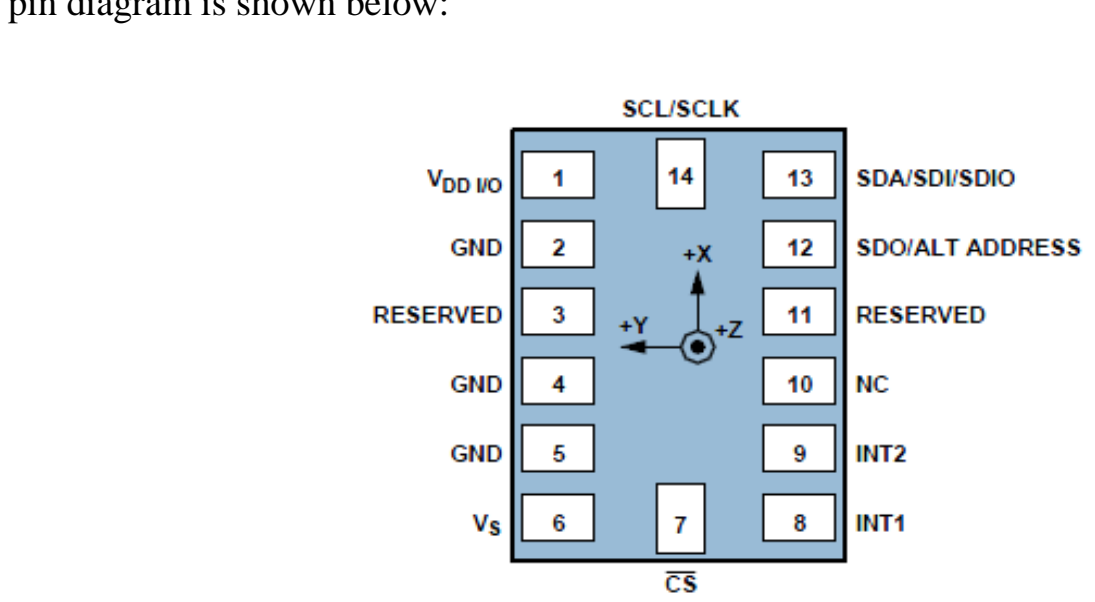

Figure 2. Pin Diagram of ADXL345

\subsection{Signal Preprocessing}

Firstly, the 13-step median filter is used to remove the noise of the sample data obtained by the acceleration acquiring module. Then, a high pass filter with the cutoff frequency of $0.5 \mathrm{~Hz}$ and a $0.8 \mathrm{~s}$ non-overlapping window superposition is adopted to eliminate the gravity factor $[12,14]$. The output is the dynamic acceleration signal to be used in the next step. After the filtering process, the classification algorithm based on 1-class SVM is adopted to extract the suspicious data. 1-class SVM algorithm is an extended version of the SVM algorithm. It uses 
the kernel function to map all the samples into a high-dimensional eigenspace to achieve the purpose of classification. In the eigenspace, 1-class SVM attempts to determine the minimum hyper spherical surface containing all the target data. This surface will be the classifier. A set of slack variables is used to control the radius of the hyper sphere and the number of samples that are outside the super sphere. This algorithm is able to extract most of the falling samples (positive samples).

For the positive sample set $X=\left\{x_{i}, i=1,2, \cdots, l\right\}, x_{i}=R^{d}$, to determine the hyper sphere (with the center of vector $a$ and the radius of $R$ ) which contains as many samples as possible through nonlinear mapping into the high-dimensional eigenspace is actually an optimization problem that can be represented by equation (1).

$$
\min _{R \in R, \xi \in R^{d}, a \in F} R^{2}+1 / v l \times \sum_{i} \xi_{i}
$$

Where,

$$
\left\|\Phi\left(x_{i}\right)-a\right\|^{2} \leq R^{2}+\xi_{i}, \xi_{i} \geq 0, i \in[1, l]
$$

Here, $F$ is an eigenspace; $\xi_{i}$ is a slack variable; $/ T v$ determines the volume of the sphere and the number of classifications that the samples outside the sphere can be classified;

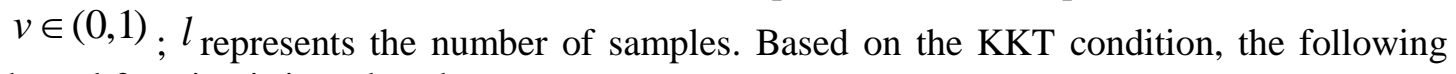
kernel function is introduced:

$$
K(x, y)=[\Phi(x) \bullet \Phi(y)]
$$

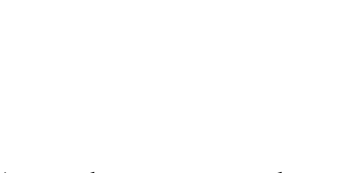

That is, the optimization equation (1) can be expressed as:

$$
\begin{gathered}
\min _{\lambda} \sum_{i, j} \lambda_{i} \lambda_{j} K\left(x_{i}, x_{j}\right)-\sum_{i} \lambda_{i} K\left(x_{i}, y_{j}\right) \\
0 \leq \lambda_{i} \leq \frac{1}{\nu}, \Delta \lambda_{i}=1
\end{gathered}
$$

Where, $\quad$ the center of the hyper sphere is:

$$
\vec{a}=\sum_{i} \lambda_{i} \Phi\left(x_{i}\right)
$$

After training, a set of support vectors can be obtained. Then, Equation (6) is used to calculate the radius $R$.

$$
R^{2}=\sum \lambda_{j} K\left(x_{i}, x_{j}\right)+K\left(x_{s}, x_{s}\right)-2 \sum_{i} \lambda_{i} K\left(x_{i}, x_{s}\right)
$$

Lere, ${ }^{x_{i}}$ is an arbitrary support vector. Its decision function is:

$$
f(x)=\operatorname{sgn}\left[R^{2}-\sum_{i, j} \lambda_{i} \lambda_{j} K\left(x_{i}, x_{j}\right)+2 \sum_{i} \lambda_{i} K\left(x_{i}, x\right)-K(x, x)\right]
$$

For all the samples, if $f(x)>0$, then this sample is a positive sample; if $f(x)<0$, then this sample is a non-positive sample. Generally, $R B F$ is used as the kernel function. 


$$
K(x, z)=\exp \left(-\|x-z\|_{2} / \sigma^{2}\right)
$$

It has been proved that it is very efficient to identify the falling actions from low-intensity daily activities, but when the activity intensity is high, it will be relatively difficult to identify the falling actions. Therefore, this algorithm is only used during the data preprocessing stage in order to extract suspicious data from the original data. By adjusting the slack variable in the experiment, it can ensure that more than $97 \%$ of the real falling sample data can be extracted.

\section{The Falling Detection Algorithm}

\subsection{The Establishment of the Action Model}

When human body is in a falling process, the acceleration, velocity and shift of the object in all directions will change. In fact, it is very difficult to comprehensively identify the falling action only in accordance with the acceleration change. The velocity $(v)$ can be obtained by conducting one time of integral of the acceleration in the time domain and the shift $(s)$ can be obtained by conducting two times of integral. This is to improye the system accuracy.

The acceleration data obtained by the acceleration sensor always contains two parts: the acceleration caused by gravity and the acceleration caused by human motion. The 3D action model is built based on the acceleration caused by human motion, and according to the three orthogonal measuring directions of the acceleration sensor, the 3D coordinate system can be established as shown in Figure 3.

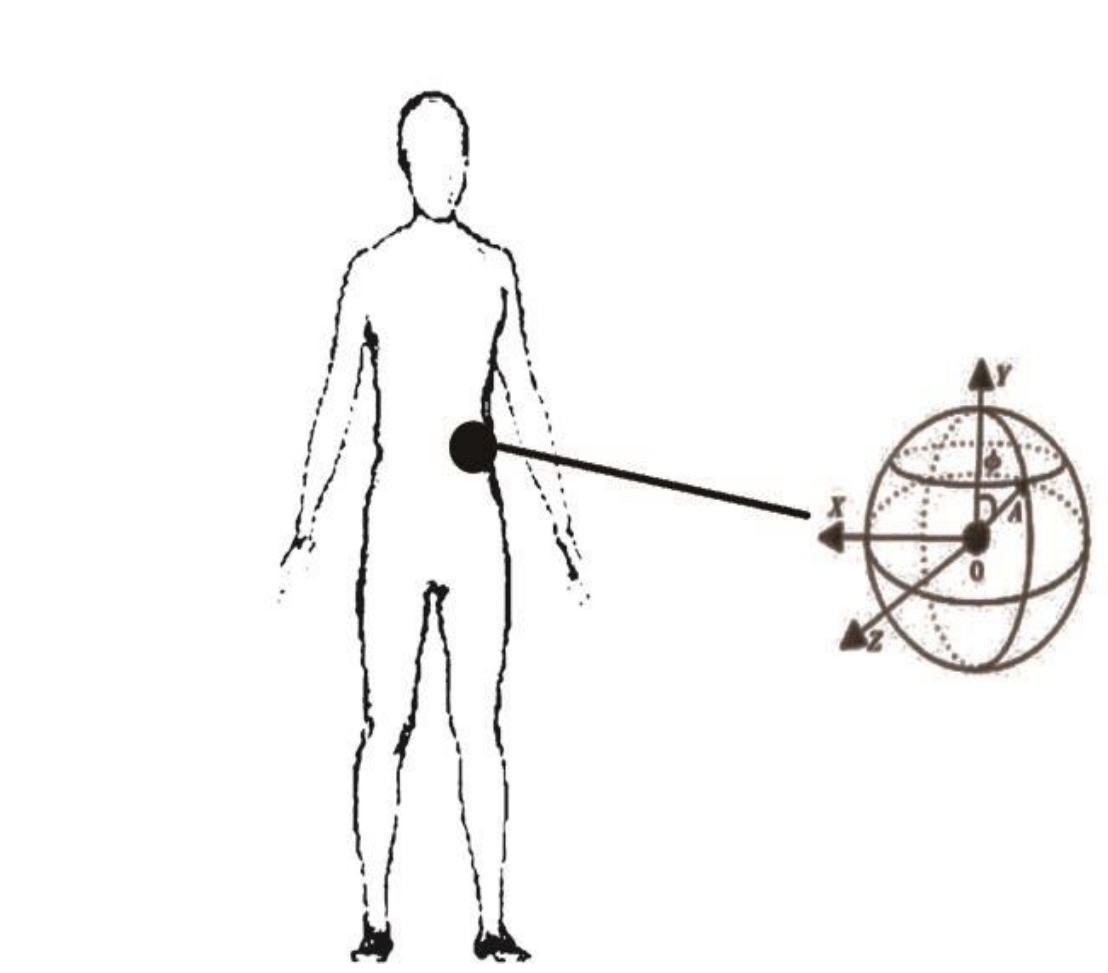

Figure 3. The Wearing Position of Fall Detection Device and Coordinate System 
If the device is correctly attached to the users and when the object is at rest or in uniform motion, the acceleration in $\mathrm{Y}$ direction should be the acceleration of gravity (g) and the acceleration in the horizontal direction should be 0 . When the object is falling, if only considering the acceleration change between the initial and the final state, the change in the vertical direction should be from $1 \mathrm{~g}$ to $0 \mathrm{~g}$, while the change in the horizontal direction ( $\mathrm{x}$ or $\mathrm{z}$ ) should be from $0 \mathrm{~g}$ to $1 \mathrm{~g}$.

Based on the coordinates, vector $\vec{A}$ can be expressed as $\vec{A}=\left(a_{x}, a_{y}, a_{z}\right)=|A| \vec{u}$, where, $|A|$ is the module of vector $\vec{A}, \vec{u}$ is the unit vector in the same direction. Then, the tilt angle of the human body $\phi$ (the angle with the direction of the acceleration of gravity.) is: $\phi=\arccos (a / g)$, where, $a$ is the static output in each axis and $g$ is the acceleration of gravity.

\subsection{The Algorithm Design}

According to the difference of energy consumption when the human body is in walking, standing and falling state, the threshold range of the energy consumption of different movements can be obtained through experiments. Hence, this study proposed the method to analyze the background data using energy consumption. The energy consumption is the integral of the square of the dynamic acceleration in the specifie time domain, which can be expressed by Equation (9):

$$
\text { EnergyExpe nditure }=a L A=a\left(\int\left|x^{2}\right| d t+\int\left|y^{2}\right| d t+\int\left|z^{2}\right| d t\right)
$$

In this paper, $a=1$; the calculation of the energy consumption considers each sampling window as the unit. According to the actual living conditions of the elderly people, the corresponding falling detection algorithm is designed. Figure 3 shows the flow chart of the overall algorithm, which describes the entire falling detection process. In this algorithm, the process from the begmning to "data transmission" is achieved at the user terminal. The whole signal preprocessing takes one sampling window as the basic unit. If suspicious data is detected durng a sampling Window, then the data of this unit will be transmitted to the background for further confirmation; otherwise, this data segment will be discarded and the system will continue the next signal. In the background data processing, the judgment is made mainly based on the analysis of energy consumption. Meanwhile, in order to improve the detection accuracy, the information of $v 、 s, \Phi$ of the human body in the specific time domain is also included as the supported data as shown in Figure 4 [15, 17]. 


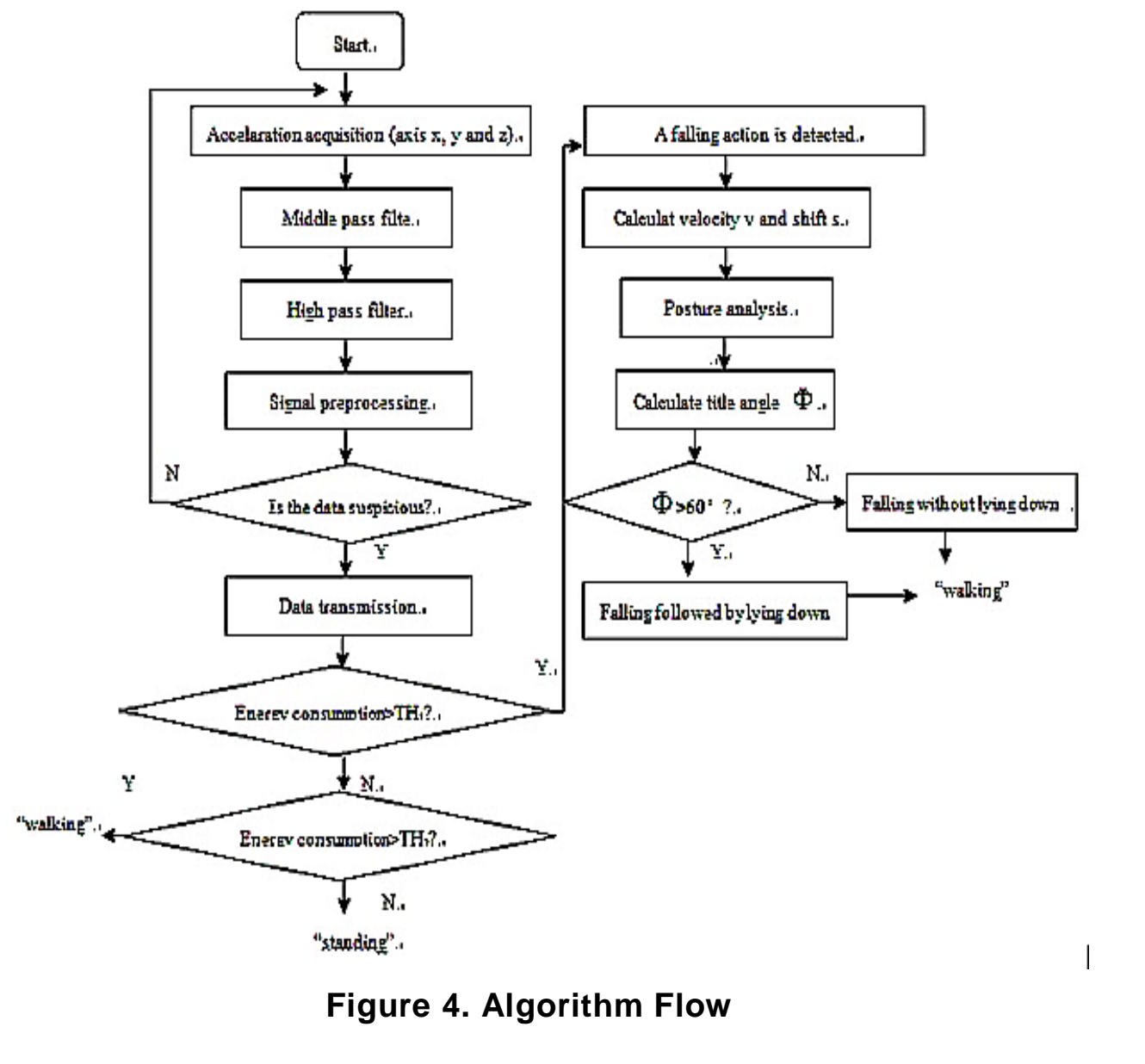

\section{Experiments and Result Analysis}

10 elderly people with the age over 60 were selected for a trial to verify the accuracy of the algorithm, but of the safety reason, they were not involved in the falling experiment. Instead, some students were invited to simulate the falling (on a protection mat). The modes of falling in the experiments are: falling forward/backward without lying down, falling forward/backward followed by lying down, falling leftward/rightward. According to these modes, a set of actions as shown in Table 1 was designed. In each experiment, the participant was required to choose some actions from this table randomly and combine with the real falling action to form a complete set of experimental action. The system firstly collected the sample data at the sampling frequency of $45 \mathrm{~Hz}$, and then, processed the data by using the proposed algorithm. Each participant was required to conduct 5 sets of experiment, and in eaen experiment, they would choose an action combination randomly from the above actions. In tota, 50 sets of experiment were completed.

The experiment results are shown in Table 1. It can be seen that the proposed falling detection alg8orithm has achieved a high accuracy. It can identify most of the falling actions, but for the falling actions without lying down and the slipping actions with a quick recovery to the balance state, there were some false judgments. 
Table 1. Experiment Data

\begin{tabular}{|c|c|c|c|c|c|}
\hline Action & $\begin{array}{l}\text { Need to } \\
\text { trigger alarm? }\end{array}$ & $\begin{array}{l}\text { No. of } \\
\text { experiment }\end{array}$ & $\begin{array}{l}\text { No. of } \\
\text { alarm }\end{array}$ & $\begin{array}{l}\text { No. of } \\
\text { non-alarm }\end{array}$ & $\begin{array}{l}\text { Accuracy } \\
\%\end{array}$ \\
\hline Standing & $\mathrm{N}$ & 50 & 0 & 50 & 100 \\
\hline Steady walking & $\mathrm{N}$ & 50 & 0 & 50 & 100 \\
\hline $\begin{array}{l}\text { Stumbling forward with } \\
\text { a quick recovery to the } \\
\text { balance state }\end{array}$ & $\mathrm{N}$ & 25 & 1 & 24 & 96 \\
\hline $\begin{array}{l}\text { Falling forward followed } \\
\text { by lying down }\end{array}$ & $\mathrm{Y}$ & 30 & 30 & 0 & 100 \\
\hline Climbing up the stairs & $\mathrm{N}$ & 15 & 0 & 15 & 100 \\
\hline $\begin{array}{l}\text { Slipping backward with } \\
\text { a quick recovery to the } \\
\text { balance state }\end{array}$ & $\mathrm{N}$ & 30 & 1 & 29 & \\
\hline $\begin{array}{l}\text { Falling backward } \\
\text { followed by lying down }\end{array}$ & $\mathrm{Y}$ & 30 & 30 & 0 & \\
\hline Climbing down the stairs & $\mathrm{N}$ & 15 & & & \\
\hline $\begin{array}{l}\text { Falling forward without } \\
\text { lying down }\end{array}$ & $\mathrm{Y}$ & 30 & & & 90 \\
\hline $\begin{array}{l}\text { Falling backward } \\
\text { without lying down }\end{array}$ & $\mathrm{Y}$ & 40 & & & 95 \\
\hline Falling rightward & $\mathrm{Y}$ & 25 & & 0 & 100 \\
\hline Falling leftward & $\mathrm{Y}$ & & & 2 & 92 \\
\hline Bedding & $\mathrm{N}$ & & & 10 & 100 \\
\hline
\end{tabular}

This study established a falling detection module based on the 3D acceleration sensor, the microprocessor and the wireless communication technology. Its validity for distinguishing daily activities and falling aetions has been proved through experiments. In the stage of data preprocessing, the data lassification allgorithm based on 1-class SVM was adopted to extract the suspicious data. Meanwhile, acreative method which is based on the difference of energy consumption in different human actions was proposed to make the final falling judgment. In addition, in order to enhance the system accuracy, the analysis about the human body posture (analysis about the velocity shift and the tilt angle of the human body) in a certain time domain was also included. However, at the user terminal, the 1-class SVM algorithm should be further optimizedin erms of original data classification (as shown in Figure 3).

\section{References}

[1] H. Hongmer, Z. Hongke and Y. Xu, "Fall Detection Using Radio Signals of Home Wireless Sensor Networks", Acta Electronica Sinica, vol. 39, no. 1, (2011), pp. 195-200.

[2] Y. Hui, Z. Xiaoyue, Z. Lixin and C. Yuzhen, "Elderly fall monitoring and remote assistance system", Computer Engineering and Applications, vol. 47, no. 35, (2011), pp. 245-248.

[3] X. Yuan and G. Xiangyang, "A Design for Fall Detection Monitoring System Based on Information Fusion of Multi-sensor", Journal of Wuhan University of Technology(Information \& Management Engineering), vol. 33, no. 5, (2011), pp. 712-716.

[4] V. Chan, P. Ray and N. Parameswaran, "Mobile e-Health monitoring: an agent-based approach", IET Commun, vol. 2, no. 2, (2008), pp. 223-230.

[5] M. Huo, M. Wu and H. Hou, "Research on Fall Detection and Daily Activity Monitoring Technologies for Older People", International Journal of Digital Content Technology and its Applications, vol. 6, no. 16, (2012), pp. 548-557.

[6] S.-W. Lee and S.-H. Song, "A Monitoring System for Assessing Life Pattern of the Elderly Living Alone", Advances in Information Sciences and Service Sciences, vol. 3, no. 7, (2011), pp. 311-323. 
[7] Y. Zhou, D. Wen, F. Yuan, J. Li and M. Li, "Research of Online Water Quality Monitoring System Based on Zigbee Network", Advances in Information Sciences and Service Sciences, vol. 4, no. 5, (2012), pp. 255-261.

[8] Y. Wang, "The Development of Wireless Personnel Positioning in Internet of Things Based on ZigBee and Sensors", International Journal of Digital Content Technology and its Applications, vol. 6, no. 12, (2012), pp. 47-54.

[9] C.-C. Lin, W.-P. Chen, S.-C. Hsieh and S.-S. Chen, "Design and Implementation of Zigbee Wireless Controller Module", Advances in Information Sciences and Service Sciences, vol. 4, no. 18, (2012), pp. 106115.

[10] O. Xi, Z. Jianyi, G. Zhe and L. Qi, "A Reputation-based Ant secure routing Protocol of Wireless Sensor Networks", International Journal of Advancements in Computing Technology, vol. 4, no. 9, (2012), pp. 1018.

[11] S. Zihao and L. Shufen, "Security Threats and Security Policy in Wireless Sensor Networks", Advances in Information Sciences and Service Sciences, vol. 4, no. 10, (2012), pp. 166-173.

[12] N. Hongcheng, T. Guifa, Z. Yang and D. Sufen, "Application research of fall detection in remote health care and supervisory system”, Microcomputer \& Its Applications, vol. 30, no. 6, (2011), pp. 76-78.

[13] Z. Ye and X. Li, "Virtual Prototyping Design and Evaluation of Ergonomic Gymnastic Based on CATIA", International Journal of Hybrid Information Technology, vol.6, no. 5, (2013), pp. 67-78.

[14] W. Liu and Y. Yan, "Application of ZigBee Wireless Sensor Network in Smart Home Sy sten?, International Journal of Advancements in Computing Technology, vol. 3, no. 5, (2011), pp. 154-160.

[15] F. Yu, M. Zong and Y. Ji, "Research and Construct of Wireless Network of Intelligen Illuminating Control System for College", International Journal of Digital Content Techmology and its Applications, vol. 6, no. 16, (2012), pp. 480-488.

[16] Z. Y and X. Li, "Model Evaluation Based on Emotional Furniture Industrial Design Elements", International Journal of Advancements in Computing Technology, vol. 22, no. 4, (2012), pp. 73-78.

[17] K. Cai and X. Liang, "Design of Wireless Acceleration Sensor Nodes Used in Mechanical Equipment Status Monitoring”, International Journal of Advancements in Computing Technology, vol. 3, no. 10, (2011), pp. 413-420.

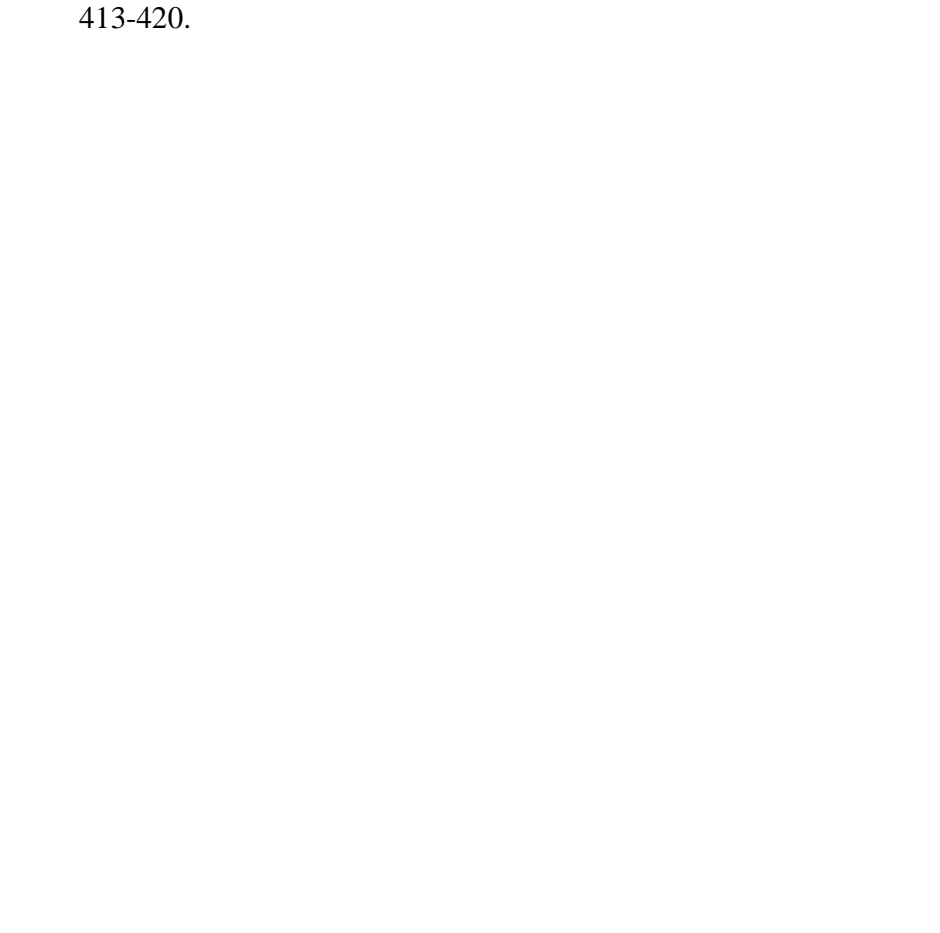


International Journal of Smart Home

Vol.8, No.1 (2014)

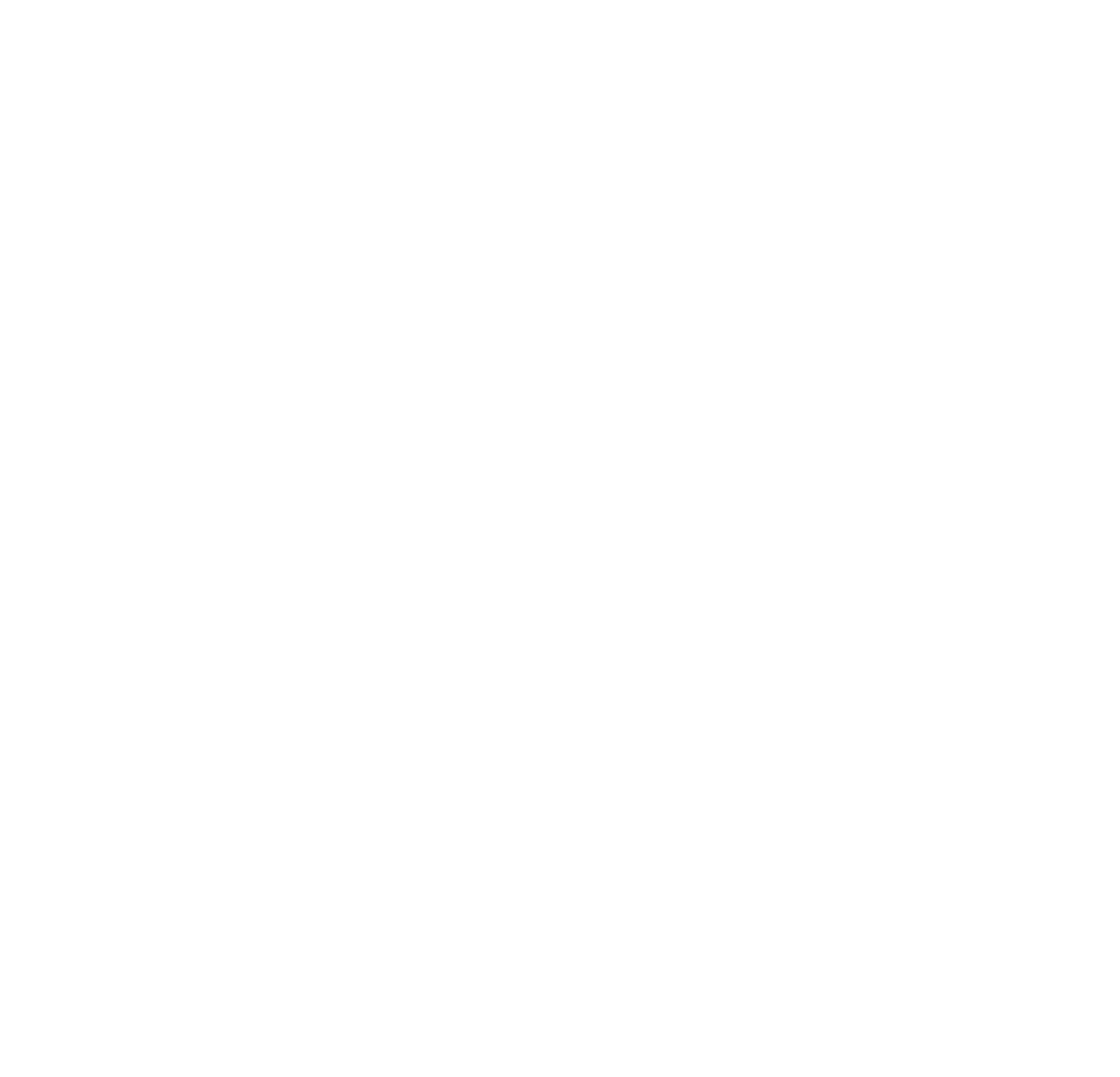

